\title{
Polarization-Insensitive Directional Couplers Based on SOI Wire Waveguides
}

\author{
Vittorio M.N. Passaro ${ }^{*}, 1$, Francesco Dell'Olio ${ }^{1}$, Branislav Timotijevic ${ }^{2}$, Goran Z. Mashanovich ${ }^{2}$ and \\ Graham T Reed ${ }^{2}$ \\ ${ }^{1}$ Photonics Research Group, Dipartimento di Elettrotecnica ed Elettronica, Politecnico di Bari, via Edoardo Orabona $n$. \\ 4, 70125 Bari, Italy \\ ${ }^{2}$ Advanced Technology Institute, University of Surrey, Daphne Jackson Building, GU27XH, Guildford, UK
}

\begin{abstract}
Optical directional couplers based on SOI-wire waveguides have been modelled by a semi-analytical approach based on the Coupled Mode Theory and Finite Element Method. The modelling is used to obtain analytically optical power at the parallel and cross ports by utilizing numerically calculated coupling coefficients. Geometrical dimensions of the couplers have been optimized to obtain a polarization-independent behaviour. The influence of non-vertical sidewalls on the coupler performance has also been addressed.
\end{abstract}

\section{INTRODUCTION}

Silicon-on-insulator (SOI) technology is emerging as a very attractive platform for active and passive photonic devices [1].

Si-wire waveguides enable fabrication of ultra-compact photonic devices and large scale of integration of optical circuits [2-3]. Moreover in Si-wire waveguides, the optical power density in waveguide core is higher by a factor of about 1000 than that in a conventional single-mode fiber. Consequently, nonlinear optical effects, including stimulated Raman scattering (SRS), stimulated Brillouin scattering (SBS), self-phase modulation (SPM), cross-phase modulation (XPM), two-photon absorption (TPA), and four-wave mixing (FWM), occur employing low input power equivalent to that typical in optical communications [4-5]. Adopting Si-wire waveguides different devices have been realized as reconfigurable optical add-drop multiplexers (ROADM) [6], micro-ring resonators [7], arrayed waveguide gratings (AWG) [8], thermo-optic switches [9], polarization splitter [10], Raman optical amplifiers [5] and wavelength converters [11].

Optical directional couplers are fundamental buildingblocks in a great variety of photonic components as switches, power combiner/dividers and multiplexers. Using two 300 $\mathrm{nm} \times 300 \mathrm{~nm}$ Si-wire waveguides spaced of $300 \mathrm{~nm}$, a directional coupler having a coupling length around $10 \mu \mathrm{m}$ has been fabricated [12].

In this paper we use a coupled mode theory (CMT)-based formalism to model directional couplers based on Si-wire waveguides. Coupling coefficients between optical modes propagating in the waveguides forming the coupler have been calculated by the full-vectorial Finite Element Method (FEM) [13]. By this model, validated by experimental results reported in the literature, the dependence of the directional

*Address correspondence to this author at the Photonics Research Group, Dipartimento di Elettrotecnica ed Elettronica, Politecnico di Bari, via Edoardo Orabona n. 4, 70125 Bari, Italy; Tel: +39 0805963850; Fax: +39 0805963410; E-mail: passaro@deemail.poliba.it coupler performance on geometrical parameters and fabrication tolerances has been investigated. The possibility to realize a polarization-independent optical coupler in Si-wire wave-guides has been also studied.

\section{SI-WIRE WAVEGUIDE BIREFRINGENCE}

The generic cross-section of each silicon wire is shown in Fig. (1a), while the directional coupler schematic is drawn in Fig. (1b). In general, the waveguide cross section can have non perfectly vertical sidewalls, due to technological tolerances. The birefringence ( $B=n_{\text {eff }}^{T E}-n_{\text {eff }}^{T M}$, i.e. the difference between the effective refractive indices of quasi TE and TM modes) of Si-wires has been investigated on the waveguides that have been recently used to demonstrate a large Free Spectral Range (FSR) optical filter [14]. By assuming a waveguide height of $290 \mathrm{~nm}, B$ was calculated by FEM as a function of waveguide width $(w)$ assuming refractive indices of $\mathrm{Si}$ - core and $\mathrm{SiO}_{2}$ - overlayer as 3.476 and 1.447 , respectively. Birefringence as a function of $w$ for waveguides with ideally vertical sidewalls is sketched in Fig. (2). Cases when the walls are inclined by $8^{\circ}$ on both sides and by $8^{\circ}$ and $10^{\circ}$ on the left and right sides, respectively, have been also investigated and shown in Fig. (2). In particular, the latter situation occurs for the fabricated structures presented in [14]. It is evident from Fig. (2) that birefringence is significantly dependent on the waveguide width in both symmetric and asymmetric cases. A small change in the waveguide width produces a birefringence change of $\Delta B / \Delta w \approx 3 \cdot 10^{-3} \mathrm{~nm}^{-1}$. In other words, waveguide birefringence can increase from zero to values around $10^{-2}$ as a consequence of a change in the waveguide width of a few of nanometers.

For vertical side walls, zero-birefringence (ZBR) condition is achieved for $w_{Z B R}=292 \mathrm{~nm}$ (see Fig. (2)). Non vertical side walls significantly perturb this condition (about $16 \%, w_{Z B R}=245 \mathrm{~nm}$ ), whereas side-wall angle asymmetry has a modest influence on the ZBR (around $-2 \%$ with respect to the symmetric non vertical case, $w_{Z B R}=239 \mathrm{~nm}$ ), as again shown in Fig. (2). 


\section{DIRECTIONAL COUPLER INVESTIGATION}

Optical directional couplers in Si-wire waveguides have been investigated by using a semi-analytic approach based

a)

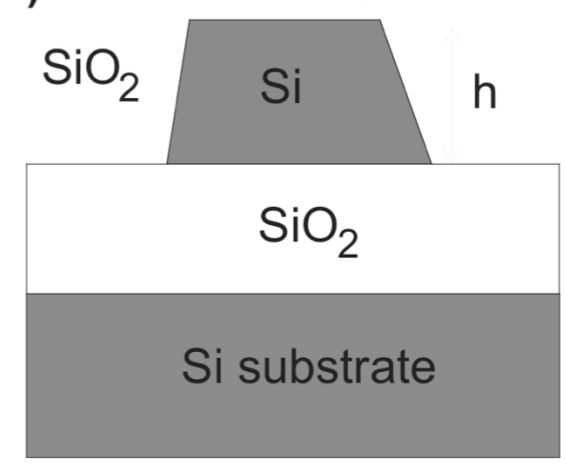

b)

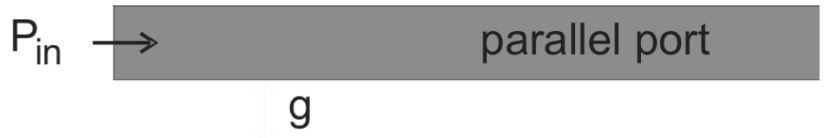

\section{cross port}

Fig. (1). (a) Profile of a generic Si - wire. (b) Schematic of modeled directional coupler.

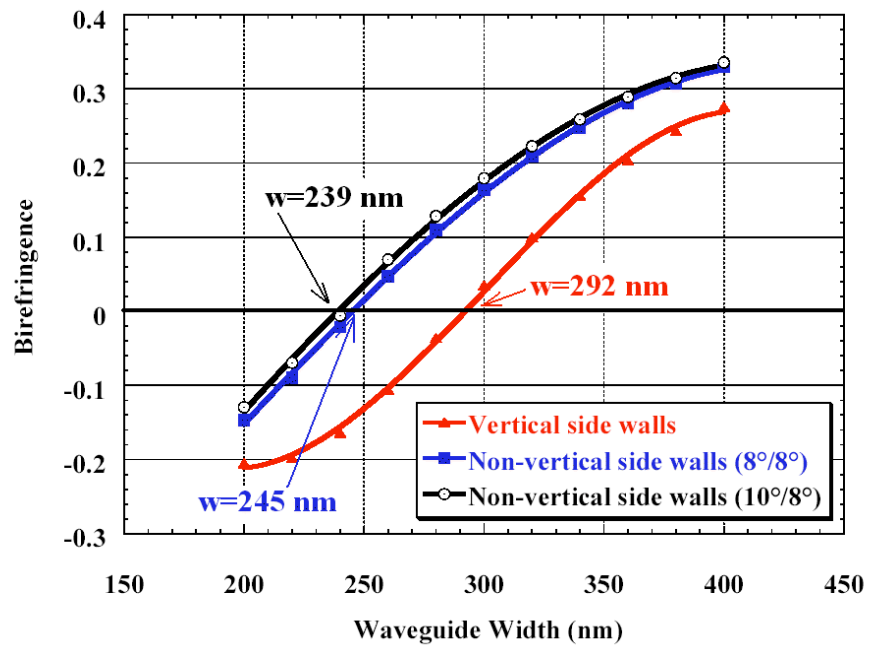

Fig. (2). Dependence of Si-wire waveguide birefringence on waveguide width $(\mathrm{h}=290 \mathrm{~nm})$.

on both FEM and CMT. According to the CMT, coupling coefficient $\kappa$ between the two optical modes propagating in the optical waveguides that form a symmetric directional coupler can be expressed as [15]:

$\kappa=\frac{1}{2}\left(\beta_{s}-\beta_{a}\right)$

where $\beta_{s}$ and $\beta_{a}$ are propagation constants of symmetric and anti-symmetric modes in the directional coupler, respectively. Using 2D full-vectorial FEM to perform modal analysis of coupler cross-section, it is possible to calculate $\beta_{s}$ and $\beta_{a}$ with high accuracy. The coupling length $L_{C}$ is related to the coupling coefficient by the following formula:
$L_{C}=\frac{\pi}{2 \kappa}$

By neglecting the losses, the normalized optical power at cross and parallel ports (see Fig. (1b)) is given by:

$$
\begin{aligned}
& \frac{P_{\text {parallel }}}{P_{\text {in }}}=\cos ^{2}\left(\frac{\pi}{2} \frac{L}{L_{C}}\right) \\
& \frac{P_{\text {cross }}}{P_{\text {in }}}=\sin ^{2}\left(\frac{\pi}{2} \frac{L}{L_{C}}\right)
\end{aligned}
$$

where $L$ is the coupler length and $P_{i n}$ the input optical power.

To validate this approach, we have simulated the coupler proposed in Ref. [12]. The dependence of normalized optical power at the parallel and cross ports as a function of $L$ for this device has been calculated, and results are sketched in Fig. (3). The modeling indicates a coupling length $L_{C}$ of 9.2 $\mu \mathrm{m}$ and $10.8 \mu \mathrm{m}$ for quasi-TM and quasi-TE modes, respectively. These results are in good agreement with those reported in [12] $\left(L_{C}=10 \mu \mathrm{m}\right.$ for quasi-TM and $L_{C}=11 \mu \mathrm{m}$ for quasi-TE mode), obtained experimentally and theoretically by a much more heavy 3D Finite-Difference Time Domain (FDTD) approach.

The coupling length dependence on distance $g$ between two Si-wire waveguides (see Fig. (1b)) has been also investigated for the waveguide widths of $300 \mathrm{~nm}$ and $340 \mathrm{~nm}$ (waveguide height is always equal to $290 \mathrm{~nm}$ ), assuming both vertical and non vertical (symmetric) side walls (as in Figs. $(4,5))$.

It can be noted from Figs. $(\mathbf{4 , 5})$ that fabrication tolerances related to non vertical side walls significantly affect $L_{C}$ dependence on the separation $g$ for quasi-TE modes, particularly for larger values of width $w$.

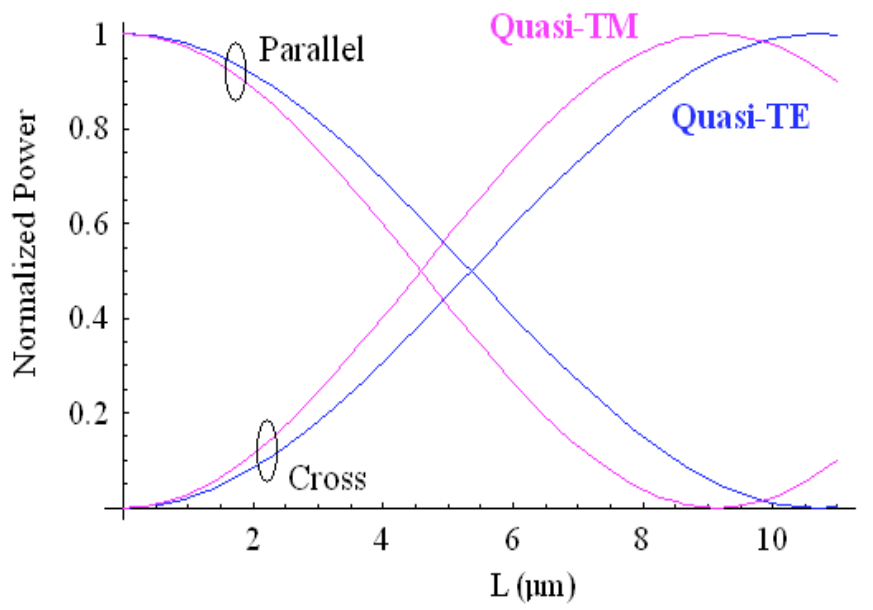

Fig. (3). Dependence of the normalized optical power at parallel and cross ports on $L$ for the device in [12].

For example, for $w=340 \mathrm{~nm}$ and $g=300 \mathrm{~nm}$, coupling length is $21 \mu \mathrm{m}$ for vertical side walls and $27 \mu \mathrm{m}$ for side wall angle of $8^{\circ}$, respectively (as in Fig. (5)). For the separation of $g=350 \mathrm{~nm}$, the difference between the two coupling lengths increases up to $12 \mu \mathrm{m}$. However, coupling length for TM polarization is almost identical for vertical and nonvertical side-walls (Fig. 5). Moreover, the coupling length strongly depends on light polarization, either for $w=300 \mathrm{~nm}$ 
or $w=340 \mathrm{~nm}$. The difference between coupling lengths related to quasi-TE and quasi-TM modes increases for larger values of the separation $g$.

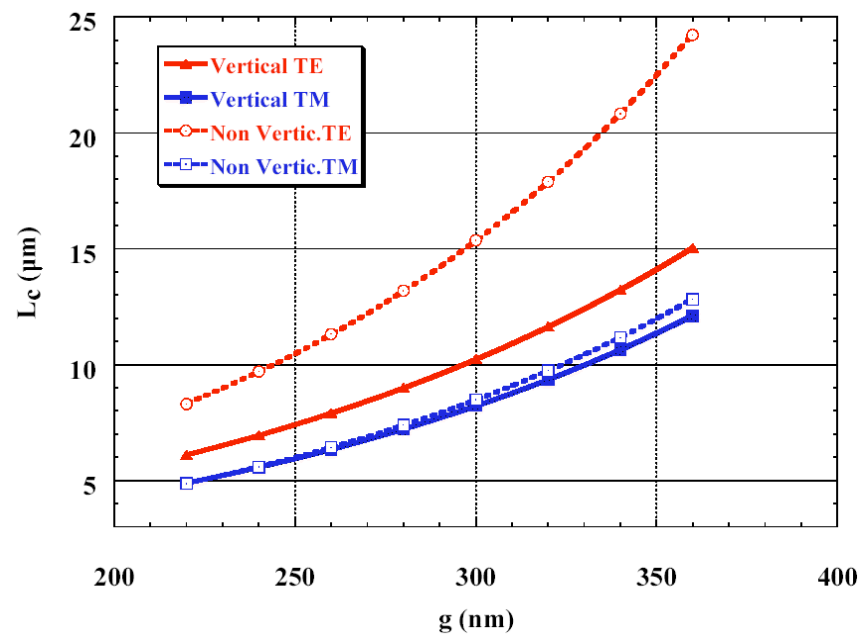

Fig. (4). Coupling length dependence on $g(h=290 \mathrm{~nm}, w=300$ $\mathrm{nm})$.

To obtain a polarization-independent optical coupler, the dependence of $L_{C}$ on $w$ has been investigated for quasi-TE and quasi-TM modes, as sketched in Fig. (6), assuming $g=$ $300 \mathrm{~nm}$ and $h=290 \mathrm{~nm}$. The polarization independence has been obtained for $w=278 \mathrm{~nm}$ and in case of vertical side walls, giving $L_{C}=6.88 \mu \mathrm{m}$. It is useful to fit the coupling length dependence on $g$ by an exponential function for best fitting of curve behavior in Figs. (4,5). Thus, it is possible to write $L_{C}$ as:

$$
L_{C}=A e^{B g}
$$

where $A$ and $B$ are fitting parameters.

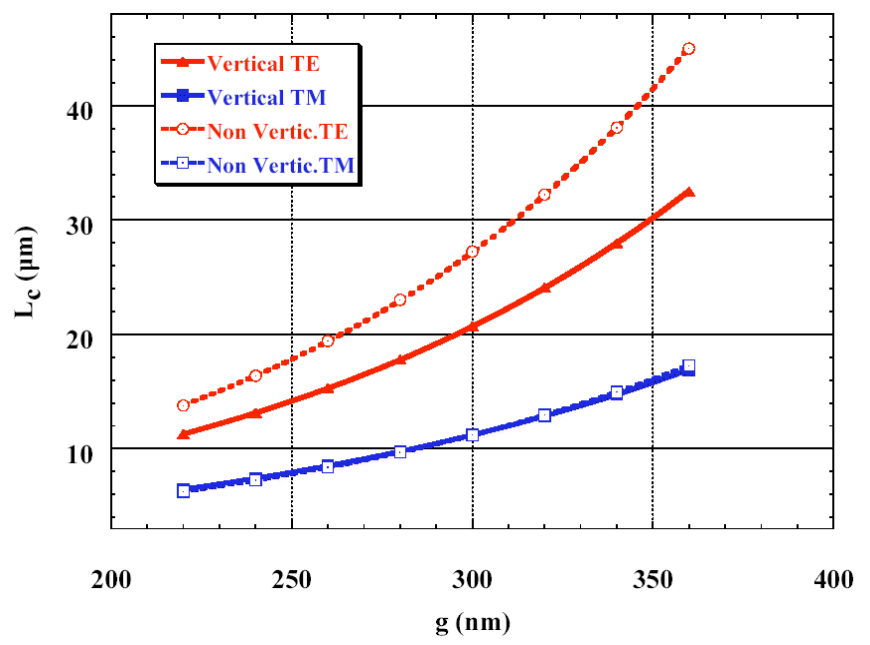

Fig. (5). Coupling length dependence on $g(h=290 \mathrm{~nm}, w=340$ $\mathrm{nm})$.

Combining equation (5) with (3) and (4), two formulas giving normalized optical power at cross and parallel ports have been derived as a function of $L$ and $g$ :

$$
\frac{P_{\text {parallel }}}{P_{\text {in }}}=\cos ^{2}\left(\frac{\pi}{2 A} L e^{-B g}\right)
$$

$\frac{P_{c r o s s}}{P_{i n}}=\sin ^{2}\left(\frac{\pi}{2 A} L e^{-B g}\right)$

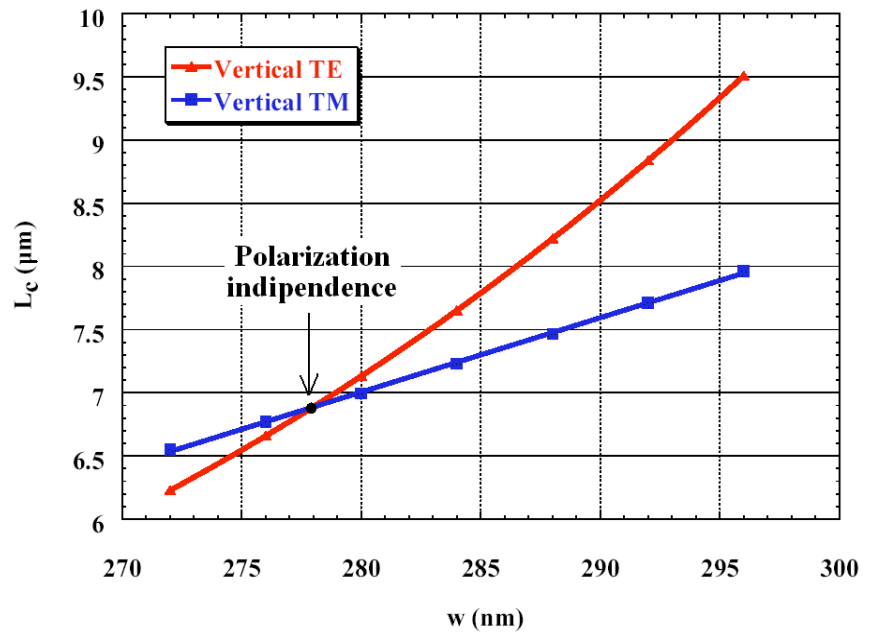

Fig. (6). Coupling length $\left(L_{c}\right)$ as a function of waveguide width (w) $(h=290 \mathrm{~nm}, g=300 \mathrm{~nm})$.

(a)

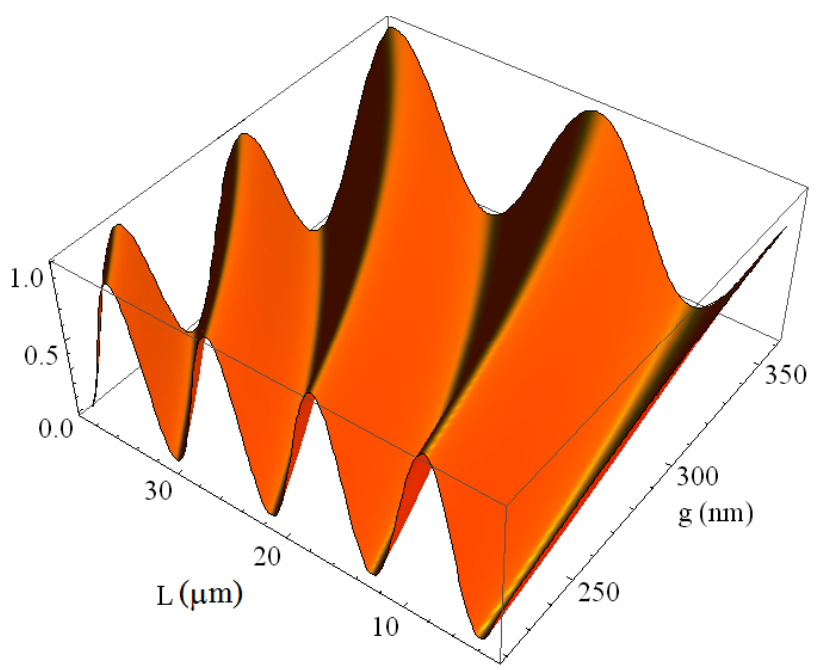

(b)

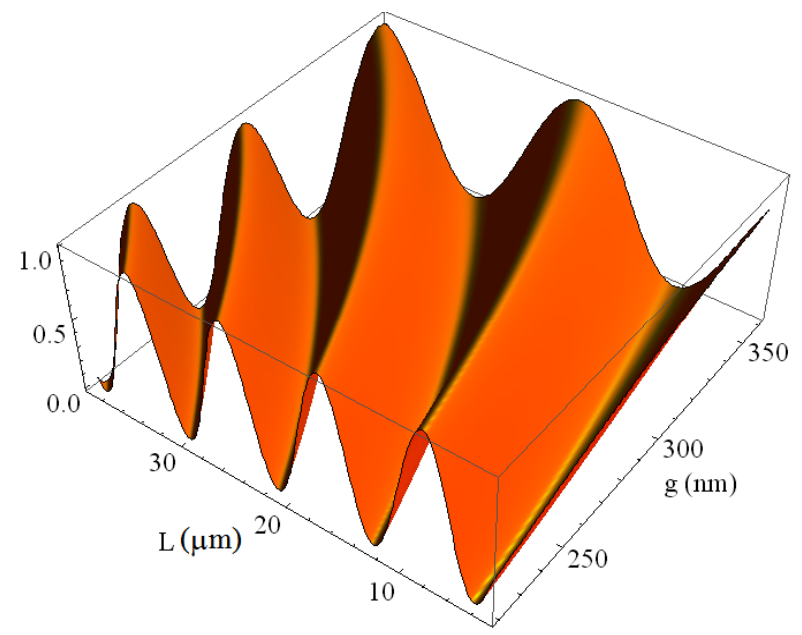

Fig. (7). Normalized optical power at parallel port plotted as a function of $g$ and $L$, for (a) quasi-TE and (b) quasi-TM modes ( $h=290$ $\mathrm{nm}, w=278 \mathrm{~nm})$. 
Considering a directional coupler based on Si-wire waveguides with height of $290 \mathrm{~nm}$ and width of $278 \mathrm{~nm}$, fitting parameters $A$ and $B$ have been calculated for both polarizations: $A=1270, B=5.637 \times 10^{-3}$ for quasi-TE mode, and $A=1121, B=6.06 \times 10^{-3}$ for quasi-TM.

Using Eq. (6) and fitting parameters, normalized optical power at parallel port is plotted as a function of $g$ and $L$, for quasi-TE (Fig. 7a) and quasi-TM mode (Fig. 7b). The two plots are practically identical. This confirms that the coupler with an optimal Si-wire waveguide having height $290 \mathrm{~nm}$ and width $278 \mathrm{~nm}$ is polarization-insensitive for $g$ ranging from 220 to $360 \mathrm{~nm}$.

\section{CONCLUSIONS}

A semi-analytical approach has been developed to optimize optical couplers based on Si-wire waveguides and to investigate the effect of non vertical sidewalls on their performance. Polarization-independence has been obtained for a $6.88 \mu \mathrm{m}$ long coupler constituted of two Si-wire waveguides with height $290 \mathrm{~nm}$ and width $278 \mathrm{~nm}$, separated by a gap of $300 \mathrm{~nm}$. The extremely short coupling length of this device makes it useful for highly compact and polarizationinsensitive power combiner or divider applications. Moreover, designed optical coupler may be very useful as a building-block for polarization-insensitive racetrack microresonators.

\section{ACKNOWLEDGEMENT}

This work was partially supported by Italian Ministry of University and Research under Interlink Project No. II04C01CDM.

\section{REFERENCES}

[1] GT Reed, AP Knights. Silicon Photonics. John Wiley \& Sons 2004

[2] Tsuchizawa T, Yamada K, Fukuda H, et al. Microphotonics devices based on silicon microfabrication technology. IEEE J Sel Top Quantum Electron 2005; 11: 232-240.

[3] W Bogaerts, R Baets, P Dumon, et al. Nanophotonic waveguides in silicon-on-insulator fabricated with CMOS technology. J Lightwave Technol 2005; 23: 401-412.

[4] H Fukuda, K Yamada, T Shoji, et al. Four-wave mixing in silicon wire waveguides. Optics Express 2005; 13: 4629-4637.

[5] RL Espinola, JI Dadap, R M Osgood, et al. Raman amplification in ultrasmall silicon-on-insulator wire waveguides. Optics Express 2004; $12: 3713-3718$.

[6] T Chu, H Yamada, S Ishida, et al. Tunable optical add-drop multiplexer based on silicon photonic wire waveguides. IEEE Photon Technol Lett 2006; 18: 1409-1411.

[7] MS Nawrocka, TL Wang, RR Panepucci. Tunable silicon resonator with wide free spectral range. Appl Phys Lett 2006; 89: 71110-171110-3.

[8] K Sasaki, F Ohno, A Motegi, et al. Arrayed waveguide grating of $70 \times 60 \mu \mathrm{m}^{2}$ size based on Si photonic wire waveguides. Electron Lett 2005; 41: 801-802.

[9] T Chu, H Yamada, S Ishida, et al. Compact $1 \times \mathrm{N}$ thermo-optic switches based on silicon photonic wire waveguides. Optics Express 2005; 13: 10109-10114

[10] H Fukuda, K Yamada, T Tsuchizawa, et al. Ultrasmall polarization splitter based on silicon wire waveguides. Optics Express 2006; 14: 12401-12408.

[11] K Yamada, H Fukuda, T Tsuchizawa, et al. All-optical efficient wavelength conversion using silicon photonic wire waveguide. IEEE Photon. Technol Lett 2006; 18: 1046-1048.

[12] H Yamada, T Chu, S Ishida, et al. Optical directional coupler based on Si-wire waveguides. IEEE Photon Technol Lett 2005; 17: 585587.

[13] Comsol Multiphysics by $\mathrm{COMSOL}^{\circ}$, Ver. 3.2., single license, 2005.

[14] BD Timotijevic, D Thomson, FY Gardes, et al. Tailoring the response and temperature characteristics of multiple serial-coupled resonators in silicon on insulator. Proc SPIE 2007; 6477: 64770B.

[15] MJ Liu. Photonic Devices. Cambridge University Press, 2005. 